\title{
Energy Conservation in a Smart Home via an Embedded
}

\section{Platform}

\author{
Rahul Sivaram \\ MIT College of Engineering \\ Ex-Serviceman Colony \\ Pune, India
}

\author{
Vikash Kumar \\ MIT College of Engineering \\ Ex-Serviceman Colony \\ Pune, India
}

\author{
Shashank Kumar \\ MIT College of Engineering \\ Ex-Serviceman Colony \\ Pune, India
}

\author{
Sahil Budhwani \\ MIT College of Engineering \\ Ex-Serviceman Colony \\ Pune, India
}

\begin{abstract}
Home automation is a popular field of application which combines the concepts of embedded systems and pervasive computing to control a living space for modern times. A smart home system aims to free up the user to carry out his or her daily tasks without worrying on the trivial aspects of personal home management, while at the same time providing the user with more control of the home than ever before. The implementation comprises of a large number of sensors which can be used to control or monitor objects distributed in three-dimensional space around the house. The sensors can be specialized in measuring temperature, humidity, pressure, light, noise, dust air, and upon intelligently computed triggers, compel the system to perform a specific task, or a set of tasks. In this project, a solution to transform a normal house into a smart, autonomous house in order to reduce the energy consumption of the household is proposed. This can be realized with the help of wired sensor networks where we control and interface with electronic appliances via a Raspberry $\mathrm{Pi}$, an embedded development platform. The embedded platform can be controlled remotely to turn switches on and off, and eventually aims to become as autonomous as possible via the help of a Bayesian Network. The base workings of the project are aimed at being as scalable as possible, to be able to potentially fit into industrial as well as office environments in the future.
\end{abstract}

Keywords: Energy Conservation, Embedded Platform, Bayesian Belief Network, Pervasive Computing.

\section{INTRODUCTION}

In the 21 st century, the advent of the internet and advances in the power and miniaturization of computing devices has made it possible to implement computers wherever we can imagine. The range of possibilities has given birth to the idea of 'Home Automation', in which computers and algorithms can be given the responsibilities of operating a household, with minimal Human interaction needed.

With the rise in popularity of cheap, embedded computers, such as the Raspberry $\mathrm{Pi}$ [8] and the Beaglebone Black computing has never been more accessible to the masses. It is proposed in this paper to take advantage of the small form factor, energy efficiency, computing power and GPIO capabilities, to interface the embedded platform with appliances and electrical sockets found in a household. These platforms can then be programmed in a way to tailor to the specific functions of the appliance it is connected to, essentially adding a level of intelligence to the otherwise "dumb" appliance. The addition of wireless modules to the embedded board adds the provision to wirelessly network the board, giving rise to the possibilities of an interconnected, intelligent home. The optimization of this wireless network could bring about a level of efficiency to a house/office space/industrial complex (depending on the appropriate scale factor) which could bring about tremendous gains in automation of menial tasks, interconnectivity of the application space, and energy usage savings.

Electricity is a precious resource which should be utilized carefully, especially in the modern era where energy requirements are rising at an alarming rate. It has become a common agony for citizens of urban spaces to face the ill effects of a poorly structured, operated and maintained energy grid. These include problems such as load shedding, and even complete blackouts. Some of the areas receive only an hour of electricity a day. As we are restricted on the front of producing electricity, this calls for a smart way to use electricity in our living spaces to create an energy efficient future. This is where our project comes in. We propose making our homes operationally seamless so that not only do we not have to worry about explicitly operating our home appliances, we can be assured the home space is functioning as efficiently and safely as possible [7]. A scenario in which every appliance turns on automatically whenever a person enters the space relevant to the context of the appliance, depending upon interlinked sensors and historical data which will act as the training set for our system. Since we will be working on limited resources we shall consider only a few appliances however the scope can be widened to accommodate various devices and so that we can obtain 
optimized metrics, and perform critical analytics and improve the learning algorithm used in the project to ensure optimal functionality [5].

The paper proposes the use of a Bayesian Network as the principle machine learning algorithm to be used. The selection of this algorithm is due to the fact it is an extremely powerful tool to model uncertainty in a situation. The algorithm does this through the data present in a Joint Probability Distribution, in which the probabilities of events are corelated, and are used to teach a machine to come to a decision about an event when there are a set of actions the machine is allowed to take in the case of the event. This principle is used to model the uncertain way a home environment operates. even the average two bedroom home, in the perspective of the appliances the home may contain, the living and usage patterns of the occupants of the home, the considerations of users of the home space who may not be permanent residents, but rather frequent visitors such as family and friends, and non-frequent users such as delivery men, semi-pertinent users such as pets, makes modeling a deterministic "trigger" mechanism for the appliances of the home quite difficult, challenging the placement of this particular computational problem in the appropriate complexity class. Using the optimally designed Bayesian Network and historical data collected from different input points into the system, it is proposed to model this "trigger" mechanism [3].

\section{RELATED WORK}

In a paper described by the Silviu Folea Automation Department Technical University of Cluj-Napoca ClujNapoca, Romania[1], the fundamental concepts which describe a framework that can be applied to develop a smart home, and can be tweaked/expanded upon as necessary. It also provides an introduction to the software "LabVIEW", which can be used to capture and interpret data from various presence sensors.

Alsheikh et al[2] in their paper take into account the framework of Wireless Sensor Networks (WSNs) and introduces pertinent topics such as issues with wireless networks, organization of WSNs, and the specifications of WSNs. The paper also discusses various machine learning algorithms (Supervised and Unsupervised) which can be applied to WSNs to optimize their functioning as well as bring a degree of autonomy to them.

A paper authored by Nancy A. Roberts for the US Air force Research Laboratory[3], introduces the implementation of the concept of a Bayesian Network to model scenarios in a smart living space for security purposes, illustrated in the paper via a simulated "Door Break-in", in which complete knowledge of the domain is unknown. More specifically, it proposes how a Bayesian Network could use historical data to attempt to predict future instances of the "Door Break-in".

A paper by Dr. Leslie Haddon [5] deals with the less technical, but nevertheless important topics regarding the aspects of home automation such as the human attitudes towards the concept of home automation, as well as how the automation of another system such as electricity production and traffic management would be applicable

\section{SYSTEM ARCHITECTURE}

As can be seen in the FIG 3.1, we propose a twofold approach to interact with the embedded platform: one is via a smartphone application which can access a dynamic webpage encoded in a scripting language such as PHP, which is hosted by a server being run in the embedded platform, implemented using the appropriate framework such as Apache or Node.js.

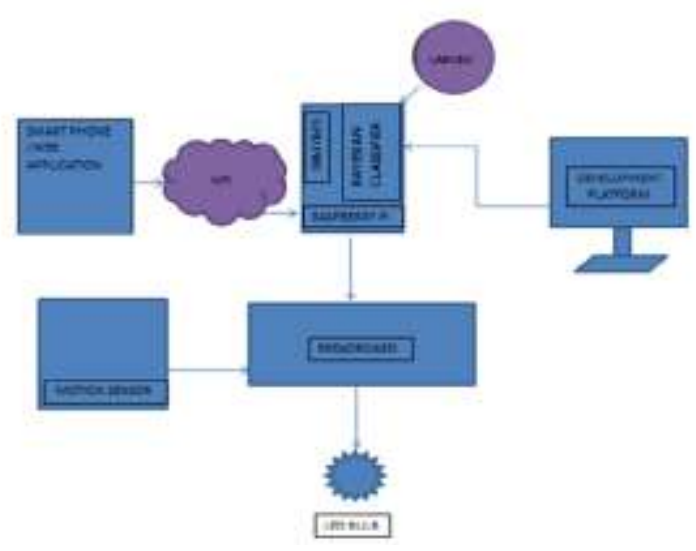

FIG 3.1: SYSTEM ARCHITECTURE

This approach demonstrates the act of explicit Human Computer Interaction (eHCI). The second approach is to have the system continuously monitor surrounding activity through a presence sensor such as a motion sensor. This would be the act of implicit Human Computer Interaction (iHCI). The system would be properly integrated through the combination of hardwiring, as well as wireless interconnectivity. Wireless connectivity is proposed to be implemented through an appropriate set of media access control and physical layer specifics, such as IEEE 802.11[4]. The appliance we consider here is an energy efficient LED bulb.

The entire system's support framework can be thought of as everything but the mobile webpage and the actual appliance being controlled.

To operate the system, the user would use a combination of the options for iHCI and eHCI which are provided. However, the ultimate goal of the proposed project is to make the system as autonomous as possible, able to predict system "trigger" events using a decision value stored in a structured database, using a probability engine. This engine would calculate probabilities based on the training data set it has access to, as well as a dynamically updated probability distribution. The attributes whose probabilities contribute to the ultimate trigger decision the system makes (whether or not to trigger), would include the quantification of common, or even uncommon, daily activities which take place in a household. However, to limit the scope of the attributes considered, due to our project only being at an early research 
stage, we propose to only consider a handful of contributing attributes for the system. It can be intuitively hypothesized that increasing the number of attributes considered would increase the accuracy of prediction of the crucial trigger decision, but consideration must be taken that some attributes may conflict with others, repealing the notion that more data is always better data, in the context of our project.

Our database design will is proposed to contain two tables: the first one is the 'input table', which will consider attributes like entry number, date, day, time range, time and also label denoting whether at that instance of time the lights are turned on or off. The second table will be the table considering the results of the probability engine, and also a final trigger decision based on how those probabilities relate to a preset threshold. The prior probability will simply by calculated by considering the duration for which appliance was on and subtracting it from one will give the probability of the second class then we can compute the likelihood that is the actual probability whether at that at this instance of time the light should be on or off.

\section{THE BAYESIAN CLASSIFIER}

Heterogeneous systems in the smart home consists of the building automation system, energy management system, fire alarm system, digital surveillance system and other network based systems. Due to the involvement of many entities and the environment being partially observable, the static decision making algorithms cannot make decisions to an acceptable degree. This calls for a smart learning algorithm which accurately speculates the triggering of appliances.

A Bayesian classifier is a supervised machine learning technique which can be employed for predictive models where the environment is highly uncertain. In the context of our proposed framework, this learning technique uses a set of labeled data where information regarding a particular instance of time i.e. that is the discretized time range is fed to the system with a class label 'yes' or 'no' denoting for which instances of time the lights should be turned on. It is based on a probalistic model where we compute the probability to trigger the appliance based on a belief network as shown in Fig 4.1. It consists of a structural model with conditional probabilities, where probability of a particular node depends upon the probability of all the incoming nodes i.e. all the parent nodes. It is only an early example of the model we will use to visualize the environment, created using the Microsoft Belief Networks Tool (MSBNx).

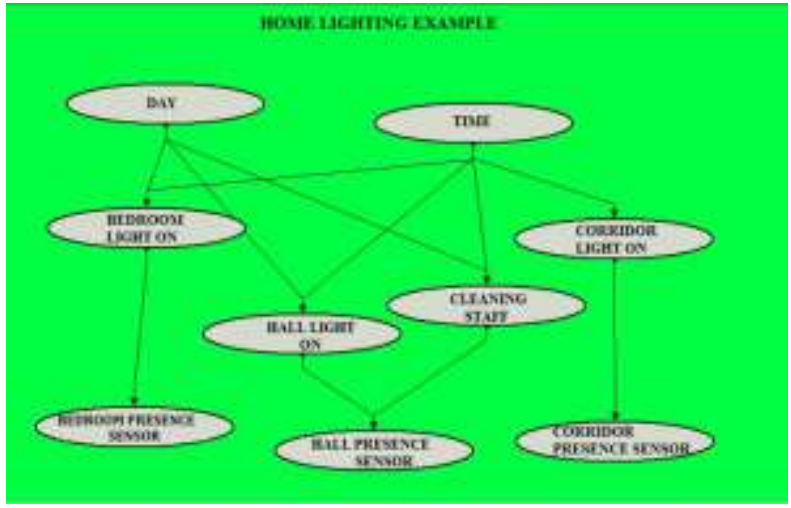

FIG 4.1: SAMPLE BELIEF NETWORK

Let us consider an example to determine in which class a set of tuples belongs based on Bayes' formula given as :

$$
P(A \mid B)=\frac{P(A) P(B \mid A)}{P(B)}
$$

Here A denotes the class which can be $\{$ yes, no $\}$

$\mathrm{B}$ denotes the tuple space $\{\mathrm{B} 1, \mathrm{~B} 2 \ldots \mathrm{Bn}\}$

$\mathrm{P}(\mathrm{A} / \mathrm{B})$ is computed by our belief network for all possible values class of $A$, and the maximum calculated value will be used for prediction via the function:

$$
\mathrm{C}=\operatorname{argmax}\left(P\left(\frac{A}{B}\right)\right)
$$

\section{CONCLUSION AND FUTURE WORK}

Home automation is a technology which is still in its infancy. Here we have provided some insight into the energy conservation and automation potential of an intelligent interconnected smart home via explicit Human Computer Interaction (eHCI) i.e. mobile application and web applications, as well as implicit Human Computer Interaction (iHCI) implemented through motion sensors. Both of these methods will present different pattern in home usage patterns, which can then be modeled to predict and optimize energy needs of a living space. There are many use cases for which we require innovative solutions to use home automation systems, such as whether a user will only conduct his or her activities in a living space for a short period of time or a long period of time. Depending on what the system can "observe" about a user's habits, the system can be programmed to take appropriate actions. The problem lies in being able to efficiently give true contextual awareness to a system. Consider a case where someone is sleeping in the room with lights turned off and another person enters the room [6]. An unintelligent system will act the way it was meant to, triggering the light, which is obviously a less than desired action. The true problem is giving the system not just awareness, but also the trait of precognition, to a certain degree. Overcoming this problem could lead to massive gains 
in the automation potential of a target environment, which could translate to an energy efficient deployment scenario.

The future scope of this project is also quite promising, as the basis of the project (the triggering mechanism) can easily be scaled to accommodate the needs of any household appliance, as well as any machine in a general sense after fine tuning the belief network which powers the trigger logic. Subsequently, if the system is implemented in a wide manner, such as with the backup of a cloud computing environment, it would be possible to expedite the training period of the system, by performing cluster analysis of people with similar patterns of living, and gain a large, more accurate, and quicker idea of how and when the system should trigger. A cloud supported framework would also let the user of the system be able to remotely monitor and operate the system, via the internet. Live energy statistics could be made available to the user to give the user information to act upon, and a user could fine tune the system further as he or she deems fit.

\section{REFERENCES}

[1] Smart Home Automation System Using Wi-Fi Low Power Devices Silviu Folea Automation Department Technical University of Cluj-Napoca Cluj-Napoca, Romania Email: silviu.folea@ aut.utcluj.ro

[2] Machine Learning in Wireless Sensor Networks: Algorithms, Strategies, and Applications Mohammad Abu Alsheikh1,2, Shaowei Lin 2, Dusit Niyato 1 and Hwee-Pink Tan 2 1School of Computer Engineering, Nanyang Technological University, Singapore 639798 2Sense and Sense-abilities Programme, Institute for Infocomm Research, Singapore 138632

[3] AFRL-IF-RS-TR-2003-25 In-House Final Technical Report February 2003 Using Bayesian Networks and Decision Theory to Model Physical Security. Nancy A. Roberts

[4] Home Automation Analysis of Current Sandeep Panigrahy, Saurabh Wahile Undergraduate Student, India, Undergraduate Student, India, International Journal of Advances in Computer Science and Technology (IJACST)

[5] Home Automation: Research Issues Dr Leslie Haddon School of Cultural and Community Studies Arts B University of Sussex Falmer, Brighton BN1 9QN UK Paper presented at the second EMTEL Workshop: The European Telecom User. November 10-11th, 1995, Amsterdam, The Netherlands

[6] http://jenson.org/easyhard/

[7] http://inhabitat.com/infographic-the-benefits-of-homeautomation-systems/

[8] https://www.raspberrypi.org/ 